\title{
Vortex quantum tunneling versus thermal activation in ultrathin superconducting nanoislands
}

\author{
W. V. Pogosov ${ }^{1,2}$ and V. R. Misko ${ }^{1}$ \\ ${ }^{1}$ Departement Fysica, Universiteit Antwerpen, Groenenborgerlaan 171, B-2020 Antwerpen, Belgium \\ ${ }^{2}$ Institute for Theoretical and Applied Electrodynamics, Russian Academy of Sciences, Izhorskaya 13, 125412, Moscow, Russia
}

(Received 15 November 2011; revised manuscript received 21 May 2012; published 6 June 2012)

\begin{abstract}
We consider two possible mechanisms for single-vortex fluctuative entry/exit through the surface barrier in ultrathin superconducting disk-shaped nanoislands made of $\mathrm{Pb}$ and consisting of just a few monoatomic layers, which can be fabricated using modern techniques. We estimate tunneling probabilities and establish criteria for the crossover between these two mechanisms depending on magnetic field and system sizes. For the case of vortex entry, quantum tunneling dominates on the major part of the temperature/flux phase diagram. For the case of vortex exit, thermal activation turns out to be more probable. This nontrivial result is due to the subtle balance between the barrier height and width, which determine rates of the thermal activation and quantum tunneling, respectively.
\end{abstract}

DOI: 10.1103/PhysRevB.85.224508

PACS number(s): 74.25.Bt, 74.25.Uv, 74.78.Na

Recent progress in miniaturization techniques has made it possible to create ultrathin quasi-two-dimensional superconducting structures, which consist of just a few monoatomic layers, and even one-layer films. In contrast to previously studied mesoscopic superconductors, ${ }^{1}$ both thermal and quantum fluctuations can be expected to be important in such extremely thin structures. ${ }^{2}$ In addition, strong confinement of condensate in lateral dimensions leads to a rather rich variety of possible behaviors. In Ref. 3, vortices in superconducting nanoislands of $\mathrm{Pb}$ with thickness of a few nanometers have been studied experimentally. It was observed that hysteresis effects are significantly weaker than expected from the theory. In another experiment, ${ }^{4}$ similar islands have been explored. The experiment has revealed a full suppression of the surface barrier ${ }^{5}$ for the single-vortex entry/exit. These results (see also Refs. 6 and 7) suggest that some kind of fluctuations of the superconducting state might be responsible for the observed features. Indeed, it was shown in Ref. 8 that the surface barrier height, under the conditions of the experiment, ${ }^{4}$ is low enough to be suppressed by thermal fluctuations.

The aim of this paper is to consider another possible mechanism of surface barrier suppression in ultrathin superconducting nanoislands, which is due to quantum fluctuations, as well as to establish a dominant mechanism, i.e., thermal activation (TA) or quantum tunneling (QT). We consider a disk-shaped superconducting island consisting of just a few monoatomic layers with radius much larger than the zerotemperature coherence length. The magnetic field is applied perpendicular to the island surface. Presence of the boundaries results in the surface barrier, which prevents vortex entry or exit. In the absence of fluctuations, the barrier for vortex entry disappears when the magnetic field reaches some critical value. The barrier for the vortex exit is suppressed at a lower magnetic field. The difference between these two fields thus reflects the significance of hysteresis effects. In the London model, which remains applicable down to low temperatures, these two critical fields can be calculated by using the approach developed by Buzdin. ${ }^{9}$ Note that macroscopic QT of Abrikosov vortices has been studied both experimentally and theoretically for the case of long current-carrying strip in zero applied field, where vortex penetration is due to the current, ${ }^{10-12}$ as well as for bulk superconductors, where energy barriers appear due to the pinning of vortices by spatial inhomogeneities ${ }^{13}$ or due to intrinsic pinning induced by the layered structure ${ }^{14}$ (for the QT of Josephson vortex, see recent papers). ${ }^{15}$ In this paper, we apply a combination of known approaches to the particular case of the ultrathin mesoscopic disks, which became experimentally testable quite recently. Apart from the interest from the viewpoint of a possible experimental examination, it is also attracting to see how strong confinement in lateral dimensions affects an interplay between quantum and thermal scenarios since it is known that probabilities of these processes are controlled by two different parameters, which are barrier width and barrier height, ${ }^{10,13,16}$ these parameters being strongly dependent on system sizes and geometry.

In order to estimate the probability of macroscopic QT of Abrikosov vortex to/from the island, we use an approach developed by Caldeira and Leggett, ${ }^{17}$ which allows one to take into account energy dissipation. For a vortex with mass $m_{v}$ in a potential $V(r)$ (to be found within the London model), the Euclidean action is given by ${ }^{17}$

$S_{E}=\int d t\left[\frac{m_{v}}{2}(\dot{r})+V(r)\right]+\frac{\eta}{4 \pi} \int d t \int d t^{\prime} \frac{\left[r(t)-r\left(t^{\prime}\right)\right]^{2}}{\left(t-t^{\prime}\right)^{2}}$.

The last term in the right-hand side (rhs) of Eq. (1), which is nonlocal, takes into account the energy dissipation. In the reciprocal space, we obtain

$$
S_{E}=\int \frac{d \omega}{2 \pi}\left\{\frac{m_{v}}{2}\left[1+\frac{\eta}{|\omega| m_{v}}\right] \omega^{2}|u(\omega)|^{2}+V(u)\right\} .
$$

It is convenient to introduce a frequency-dependent effective mass as $m_{\mathrm{eff}}(\omega)=m_{v}+\eta /|\omega|$. Note that it is shown in the following that mass $m_{v}$ is much smaller than the contribution from the viscosity.

In principle, one can use Eq. (2) to find optimal $u(\omega)$ exactly. However, it is known that quite accurate results for the effective action can be obtained by using dimensional estimates ${ }^{13}$ (in addition, we also use the method of Ref. 14). Following Refs. 10 and 13, we find the characteristic tunneling frequency $\omega_{0}$ from the balance of kinetic and potential energies as

$$
m_{\mathrm{eff}}\left(\omega_{0}\right) l_{b}^{2} \omega_{0}^{2}=V_{b},
$$


where $V_{b}$ and $l_{b}$ are barrier height and width, respectively. Equation (3) is a quadratic equation, from which $\omega_{0}$ can be found. In the viscous limit $\left(m_{v} V_{b} \ll \eta^{2} l_{b}^{2}\right)$, the solution reads as

$$
\omega_{0} \approx \frac{V_{b}}{\eta l_{b}^{2}}\left[1-\frac{m_{v} V_{b}}{\eta^{2} l_{b}^{2}}\right],
$$

where we have kept a linear term. It will be seen in the following that the dissipative regime is indeed relevant to the situation we consider here. Using Eqs. (2) and (4), we find the action as

$$
S_{E} \approx 2 \eta l_{b}^{2}\left(1+\frac{m V_{b}}{\eta^{2} l_{b}^{2}}\right) .
$$

Note that in the case of quadric-plus-cubic potential, which was solved exactly in Ref. 16, there appears a prefactor $\pi / 2$ instead of 2 in the dominant term of the rhs of Eq. (5), which demonstrates a high accuracy of the method of dimensional estimates.

The viscosity coefficient for the quasi-two-dimensional superconductor, in the case of Ohmic dissipation, is

$$
\eta=\frac{\Phi_{0}^{2}}{2 \pi \xi(T)^{2}} \frac{1}{R_{N}},
$$

where $R_{N}$ is a two-dimensional resistance, which depends on island thickness $d$ as $^{11} R_{N}=R_{0} d_{0} / d$, where $d_{0}$ is the thickness of one monolayer. According to Ref. $11, R_{0}$ is nearly equal to $6 \mathrm{kOhm}$ for $\mathrm{Pb}$.

The mass of a vortex can be estimated using the wellknown expression by Suhl. ${ }^{18}$ It includes two contributions: (i) due to the kinetic energy of the vortex core and (ii) due to the electromagnetic energy. In Ref. 19, another contribution was found, which was attributed to the shear deformations of the crystal lattice. This additional contribution is typically of the same order as those in Ref. 18, so that it does not violate viscous-limit conditions. These conclusions apply to the dirty-limit regime, which is realized for superconducting nanostructures placed on a disordered substrate, as relevant for the experiments. ${ }^{3,4}$ In contrast, in the clean limit, one has to take into account a much larger vortex mass arising from the quantization of the electron states in the vortex core, ${ }^{20}$ which may depend on the frequency of the external drive. In the dirty limit, however, it becomes of the order of Suhl mass. ${ }^{19,21,22}$

The probability of QT, within exponential accuracy, is $P_{\mathrm{QT}} \sim \exp \left(-S_{E} / \hbar\right)$, while the probability of TA is $P_{\mathrm{TA}} \sim$ $\exp \left(-V_{b} / k_{B} T\right)$. The effective action, in the dissipative limit, is mainly due to the width of the barrier, as follows from Eq. (5) (see also Refs. 10 and 13). Thus, the probability of QT is controlled by barrier width. At the same time, the probability of TA is determined predominantly by the barrier height. In the following, we show that the subtle balance between the two characteristics of the barrier leads to a rather rich behavior of the system we here study.

In the London model, the energy as a function of the vortex position $\rho$ can be found analytically. ${ }^{9}$ For a disk of radius $R$ much smaller than the effective penetration depth $\lambda_{\perp}=$ $\lambda(T)^{2} / d$, the position-dependent part of the energy reads as ${ }^{9}$

$$
U(\rho)=\frac{\Phi_{0}^{2} d}{8 \pi^{2} \lambda(T)^{2}}\left\{\ln \frac{R^{2}-\rho^{2}}{R \xi(T)}+\frac{\Phi}{\Phi_{0}}\left(\frac{\rho^{2}}{R^{2}}\right)\right\},
$$

where $\Phi$ is a magnetic flux through the disk.
We consider a superconducting island placed on a disordered substrate. ${ }^{3,4}$ Thus, the diffusive regime is realized with the mean-free path nearly equal to $2 d$. It was shown experimentally in Ref. 23 that the Ginzburg-Landau theory remains applicable for such extremely thin films. For $\lambda(T)$ and $\xi(T)$, we use the diffusive-limit expressions given by

$$
\lambda(T)=0.6 \lambda_{0} \sqrt{\frac{\xi_{0} / 2 d}{1-T / T_{c}}}, \quad \xi(T)=\sqrt{\frac{2 \xi_{0} d}{1-T / T_{c}}},
$$

where bulk $\lambda_{0}=40 \mathrm{~nm}$ and $\xi_{0}=80 \mathrm{~nm}$ for $\mathrm{Pb}$. For the dependence of $T_{c}$ on $d$, we utilize the expression deduced from the experiments on few-monolayer $\mathrm{Pb}$ films: ${ }^{23}$

$$
T_{c}(d)=T_{c 0}\left(1-\frac{d_{c}}{d}\right),
$$

where $T_{c 0}=7.2 \mathrm{~K}$ is the critical temperature of $\mathrm{Pb}$ in a bulk, while the "critical thickness" $d_{c}$ corresponds nearly to $1.5 \mathrm{ML}$.

It is easy to show from Eq. (7) that the position of the saddle point, which separates vortex-free and one-vortex states, is

$$
\rho_{c}=R\left(\frac{\Phi-\Phi_{0}}{\Phi}\right)^{1 / 2} \text {. }
$$

The condition for the vortex entry can be obtained by equating $\rho_{c}$ to $R-\xi(T)$. After simple algebra, we obtain the critical flux $\Phi_{\text {in }}$, at which barrier for vortex entry disappears:

$$
\frac{\Phi_{\text {in }}}{\Phi_{0}}=\frac{R}{2 \xi(T)} \frac{1}{1-\frac{\xi(T)}{2 R}} .
$$

When magnetic flux is lower than $\Phi_{\text {in }}$, the barrier height $U_{\text {in }}$ for the vortex entry is nonzero. It can be calculated as the difference in energies in a saddle-point configuration and at $\rho=R-\xi(T)$ :

$$
\begin{aligned}
U_{\text {in }}= & \frac{\Phi_{0}^{2} d}{8 \pi^{2} \lambda(T)^{2}}\left\{\ln \frac{R}{\xi(T)\left(2-\frac{\xi(T)}{R}\right)}-\ln \frac{\Phi}{\Phi_{0}}\right. \\
& \left.+\frac{\Phi}{\Phi_{0}}\left(\frac{2 \xi(T)}{R}-\frac{\xi(T)^{2}}{R^{2}}\right)-1\right\} .
\end{aligned}
$$

The condition for vortex exit is $\rho_{c}=0$, which immediately leads to the critical flux $\Phi_{\text {out }}=\Phi_{0}$. When $\Phi>\Phi_{\text {out }}$, the barrier height $U_{\text {out }}$ for vortex exit is nonzero. It can be found as a difference of energies in a saddle-point state and at $\rho=0$ :

$$
U_{\text {out }}=\frac{\Phi_{0}^{2} d}{8 \pi^{2} \lambda(T)^{2}}\left\{\left[\frac{\Phi}{\Phi_{0}}-1\right]-\ln \frac{\Phi}{\Phi_{0}}\right\} .
$$

Thus, it is possible to find analytically barrier heights both for vortex entry and exit. However, it is not possible to find the barrier widths analytically since it amounts solving transcendental equations. Namely, the barrier width for vortex entry is given by the difference in two values of $\rho$, the first one being $R-\xi(T)$, while the second one corresponding to another solution of equation $U(\rho)=U[R-\xi(T)]$. Similarly, the barrier width for vortex exit is given by a nonzero value of $\rho$, at which $U(\rho)=U(0)$. The above two equations are then solved numerically.

Let us focus on the case of an island with $R=120 \mathrm{~nm}$ and $d=1 \mathrm{~nm}$. These are realistic parameters which are available in modern experiments. The disk radius in terms of $\xi(0)$ is 


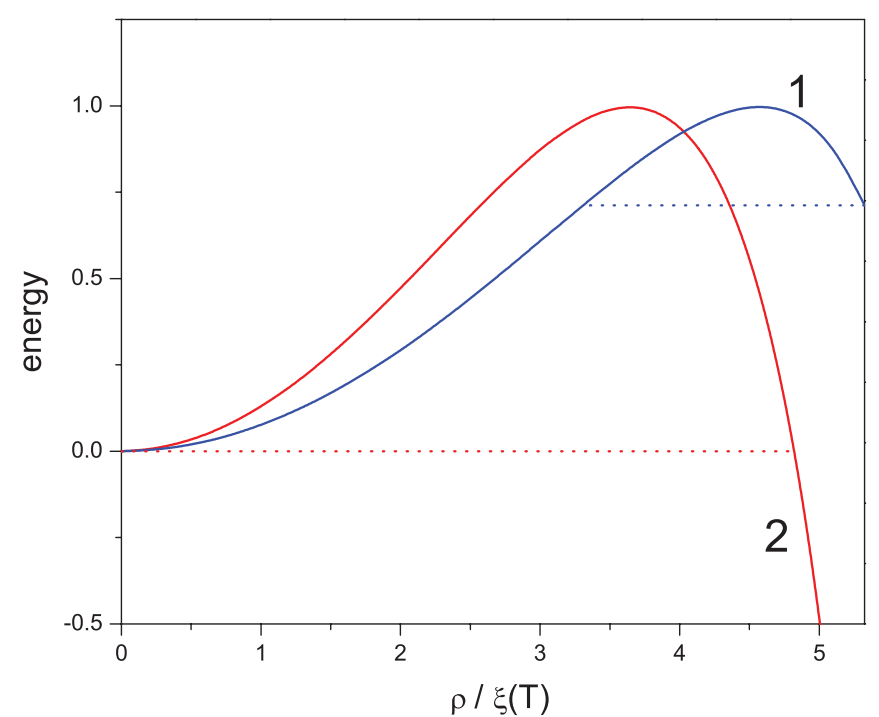

FIG. 1. (Color online) Typical curves for the energy of the system as a function of the vortex position in a superconducting island of radius $120 \mathrm{~nm}$ and thickness of $1 \mathrm{~nm}$. Curve 1 describes the barrier for vortex entry at $\Phi=2.1 \Phi_{0}$ and $T=0.6 T_{c}$. Curve 2 corresponds to the barrier for vortex exit at $\Phi=1.5 \Phi_{0}$ and $T=0.6 T_{c}$. The energies are normalized by their values at saddle points. Dotted lines are guides for eyes used to depict barrier widths.

10 , while $d$ is nearly $3 \mathrm{ML}$. This leads to $T_{c}=3.6 \mathrm{~K}$. In Fig. 1, we show typical energy profiles for vortex entry/exit. It is quite remarkable that the width of the barrier for the entry is generally much smaller than that for the exit. Since the probability of QT is controlled by the square of the barrier width, we may expect that such an asymmetry can provoke thermal escape of a vortex from the island to be more probable than QT.

Now, we are going to address two issues. First, we would like to understand if TA and/or QT is realistic for the system we consider. Second, we want to establish which mechanism is dominant depending on $T$ and $\Phi$.

Our calculations show that exponents for both QT and TA turn out to be $\sim 10-100$ almost for the whole phase diagram. We believe that values close to 10 or even several times larger are sufficient for the vortex to overcome the barrier: Indeed, it was shown in Refs. 8, 24, and 25, by evaluating the pre-exponential factors, that similar values of the exponent result in observable rates for TA for both bulk and nanosized superconductors. In addition, we ensure that we are indeed in the dissipative limit since $m_{v} V_{b} / \eta^{2} l_{b}^{2}$ is several orders of magnitude smaller than 1 .

Our results are summarized in Fig. 2. Curve 1 shows $\Phi_{\text {in }}$ as a function of $T$. Curve 2 represents $\Phi_{\text {out }}$. Dashed curve 3 gives $\Phi$, at which the energies of the state with vortex at the disk center and at $\rho=R-\xi(T)$ are equal. For the domain on the phase diagram, which is between curves 3 and 1, vortex entry is energetically favorable. For the region between curves 3 and 2, vortex exit is preferable.

Curve 4 separates two domains: below this curve change of the topological charge is more probable through thermal fluctuations, while above this curve quantum fluctuations dominate. We denote the corresponding temperature as $T_{0}$, while $k_{B} T_{0}=(\hbar / 2 \eta)\left(V_{b} / l_{b}^{2}\right)$. For the domain between curves

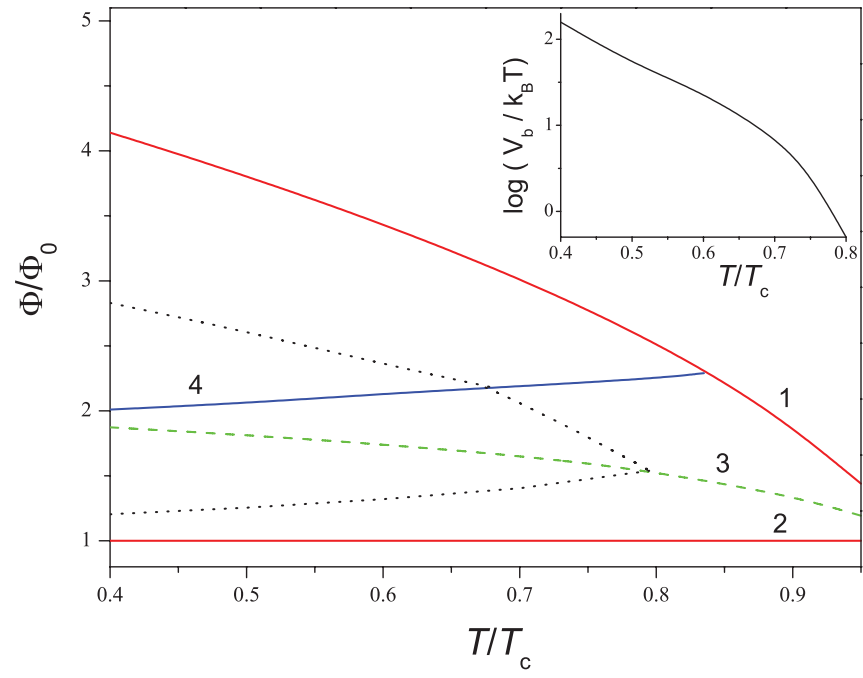

FIG. 2. (Color online) The "phase diagram" "magnetic field vs temperature" for the island (see the text). The inset shows the dependence of the decimal logarithm of $S_{E} / \hbar$ on $T / T_{c}$ along curve 4 .

4 and 1, QT of the vortex into the disk is more probable than TA. We see that the width of this region expands with decreasing $T$, which is reasonable. For the region confined between curves 1,3 , and 4, TA of vortex into the disk is more probable. It is remarkable that this scenario survives down to rather low $T$. The domain between curves 2 and 3 corresponds to the vortex escape from the island. In contrast to the case of vortex exit, it is fully determined by the thermal mechanism, even at relatively low $T$. This is due to the fact that barrier width for exit is quite large and is controlled mostly by the disk radius, while the one for the entry is mainly determined by $\xi(T)$ (see Fig. 1). The inset in Fig. 2 shows the dependence of $V_{b} / k_{B} T$ on $T / T_{c}$ along curve 4 , where it is equal to $S_{E} / \hbar$. It is also of interest that curve 4 is actually oriented nearly horizontally in this "phase diagram." It crosses curve 1, which corresponds to the threshold for the vortex entry in the absence of fluctuations, at $T \approx 0.85 T_{c}$. This implies that, along curve 1 , QT remains more probable up to quite high $T$.

Finally, two dotted lines in Fig. 2 depict borders of the regions, close to the threshold values of $\Phi$, where the exponent for the dominant mechanism of vortex entry/exit is lower than 10 , so that a change in the system's topological charge becomes highly probable. These lines can be therefore interpreted as those corresponding to the disappearance of the barrier. They intersect in the point on the curve 3 , where both barriers are suppressed, so that the vortex exit and entrance must become nearly reversible.

In this paper, we used the method of dimensional estimates, based on manipulations with two main parameters of the barrier, namely, barrier width and height. In order to provide an additional support for our results, we also applied the approach of Ref. 14, which was initially developed to address quantum creep in layered high- $T_{c}$ materials. Within this method, the effective action is first calculated in the high-temperature regime, which is purely due to TA. Then, the crossover temperature $T_{0}$ is found by solving the equation of motion taking into account only the first Fourier harmonic as a 
correction to the static solution. For $T<T_{0}$, the action is estimated, again, by using the TA expression but at $T=T_{0}$. We found that the results of the method used here turn out to be quite similar to the results of this approach. For instance, $T_{0}$ can be obtained from Eq. (25) of Ref. 14 by dropping the irrelevant elastic term, as $k_{B} T_{0}=-\hbar U^{\prime \prime}\left(\rho_{c}\right) / 2 \pi \eta$. Since $U^{\prime \prime}\left(\rho_{c}\right) \approx-V_{b} / l_{b}^{2}$, we arrive at the similar result for $T_{0}$. By evaluating $U^{\prime \prime}\left(\rho_{c}\right)$, one can also easily show that $k_{B} T_{0} \sim$ $\left(\Phi-\Phi_{0}\right)$, in agreement with curve 4 in Fig. 2 . The difference in numerical prefactors of $T_{0}$, as found by two approaches, is essentially compensated by a very sharp slope of $T_{0}$ as a function of $\Phi$.

The important question is how the predicted features can be tested experimentally. It is possible to detect presence of a vortex in a disk by using STM methods, as already has been done in Refs. 3 and 4 for smaller and thicker disks. In particular, Ref. 3 studied the difference between magnetic fields for which vortex entry and exit occur. It was shown that this quantity is significantly smaller than it could be expected from the theory when disregarding fluctuations. In our point of view, it is attractive to address the temperature dependence for average fields of vortex entry and exit. One may assume that the barrier becomes suppressed when the exponent approaches some critical value, the reasonable estimate being 10. As seen from Fig. 2, in the absence of fluctuations, the flux of vortex exit must be independent on $T$, while in the presence of fluctuations, it must grow with increasing $T$. At low $T$, the corresponding value of magnetic flux must be close to $\Phi_{\text {out }}$. By expanding the rhs of Eqs. (10), (12), and (13) in powers of $\Phi-\Phi_{\text {out }}$, one can easily show that the width of the fluctuative region where thermal fluctuations are dominant varies with temperature as $\sim \sqrt{T}$. The same applies to the case of vortex entry. Contrarily, when quantum fluctuations are dominant, the width of the fluctuative region is nearly $T$ independent [except for a rather weak dependence through $\xi(T)$ and $\lambda(T)$ ], so that it tends to a constant at $T=0$. Hence, by analyzing the low- $T$ behavior, one might distinguish between two different mechanisms of barrier suppression through fluctuations. For the vortex exit, we predict a crossover between QT and TA (see the upper dotted curve in Fig. 2). In this case, the slopes of the dependencies of the effective critical flux on $T$, which are nearly linear, must be different for low and high $T$.

Perhaps, a more elaborate method to study TA of a single vortex in ultrathin islands has been proposed very recently in Ref. 26, where it was concluded that islands of this kind provide an ideal platform to study such phenomena. It was suggested to change the applied field periodically and slowly and to measure not only the average fields for vortex entry/exit, but also their distributions. The distribution profile must broaden with the increase of $T$ in the case of TA. This feature provides an additional tool to distinguish between TA and QT scenarios.

In summary, we have studied the possibility of both the macroscopic quantum tunneling and thermal activation of Abrikosov vortex through the surface barrier in ultrathin superconducting islands made of $\mathrm{Pb}$ and consisting of just few monoatomic layers. Such islands can be fabricated and studied using modern techniques. We have found that the barrier for vortex entry, as well as for the exit, can be suppressed in this system, even not necessarily in the immediate vicinity of threshold values of magnetic flux in the absence of fluctuations. The dominant mechanism of vortex fluctuative entry/exit is determined by the interplay between the barrier height and width, which are the main quantities defining probabilities of thermal activation and quantum tunneling in a dissipative limit, respectively. A "phase diagram" of the island in the plane of magnetic flux and temperature was constructed.

This work was supported by the "Odysseus" Program of the Flemish Government and the Flemish Science Foundation (FWO-V1). W.V.P. acknowledges numerous discussions with A. O. Sboychakov and the support from the Dynasty Foundation, the RFBR (Project No. 12-02-00339), and RFBR-CNRS programme (Project No. 12-02-91055).

\footnotetext{
${ }^{1}$ A. K. Geim, S. V. Dubonos, I. V. Grigorieva, K. S. Novoselov, F. M. Peeters, and V. A. Schweigert, Nature (London) 407, 55 (2000); I. V. Grigorieva, W. Escoffier, V. R. Misko, B. J. Baelus, F. M. Peeters, L. Y. Vinnikov, and S. V. Dubonos, Phys. Rev. Lett. 99, 147003 (2007); D. S. Golubović, W. V. Pogosov, M. Morelle, and V. V. Moshchalkov, ibid. 92, 177904 (2004).

${ }^{2}$ A. Larkin and A. Varlamov, Theory of Fluctuations in Superconductors (Oxford University Press, Oxford, 2004).

${ }^{3}$ T. Nishio, T. An, A. Nomura, K. Miyachi, T. Eguchi, H. Sakata, S. Lin, N. Hayashi, N. Nakai, M. Machida, and Y. Hasegawa, Phys. Rev. Lett. 101, 167001 (2008).

${ }^{4}$ T. Cren, D. Fokin, F. Debontridder, V. Dubost, and D. Roditchev, Phys. Rev. Lett. 102, 127005 (2009).

${ }^{5}$ C. P. Bean and D. J. Livingston, Phys. Rev. Lett. 12, 14 (1964).

${ }^{6}$ H. Bartolf, A. Engel, A. Schilling, K. Il'in, M. Siegel, H.-W. Hubers, and A. Semenov, Phys. Rev. B 81, 024502 (2010).

${ }^{7}$ L. N. Bulaevskii, M. J. Graf, C. D. Batista, and V. G. Kogan, Phys. Rev. B 83, 144526 (2011).

${ }^{8}$ W. V. Pogosov, Phys. Rev. B 81, 184517 (2010).
}

${ }^{9}$ A. I. Buzdin and J. P. Brison, Phys. Lett. A 196, 267 (1994).

${ }^{10}$ L. I. Glazman and N. Ya. Fogel, Fiz. Nizk. Temp. 10, 95 (1984) [Sov. J. Low Temp. Phys. 10, 51 (1984)].

${ }^{11}$ Y. Liu, D. B. Haviland, L. I. Glazman, and A. M. Goldman, Phys. Rev. Lett. 68, 2224 (1992).

${ }^{12}$ F. Tafuri, J. R. Kirtley, D. Born, D. Stornaiuolo, P. G. Medaglia, P. Origiani, G. Belestrino, and V. G. Kogan, Europhys. Lett. 73, 948 (2006).

${ }^{13}$ G. Blatter, M. V. Feigel'man, V. B. Geshkenbein, A. I. Larkin, and V. M. Vinokur, Rev. Mod. Phys. 66, 1125 (1994).

${ }^{14}$ B. I. Ivlev, Yu. N. Ovchinnikov, and R. S. Thompson, Phys. Rev. B 44, 7023 (1991).

${ }^{15}$ E. Goldobin, K. Vogel, O. Crasser, R. Walser, W. P. Schleich, D. Koelle, and R. Kleiner, Phys. Rev. B 72, 054527 (2005); A. O. Sboychakov, S. Savelev, A. L. Rakhmanov, and F. Nori, Phys. Rev. Lett. 104, 190602 (2010).

${ }^{16}$ A. I. Larkin and Yu. N. Ovchinnikov, Pis'ma v ZhETF 37, 322 (1983) [JETP Lett. 37, 382 (1983)].

${ }^{17}$ A. O. Caldeira and A. J. Leggett, Ann. Phys. (NY) 149, 374 (1983). 
${ }^{18}$ H. Suhl, Phys. Rev. Lett. 14, 226 (1965).

${ }^{19}$ E. M. Chudnovsky and A. B. Kuklov, Phys. Rev. Lett. 91, 067004 (2003).

${ }^{20}$ N. B. Kopnin, Pis'ma ZhETF 27, 417 (1978) [JETP Lett. 27, 390 (1978)]; N. B. Kopnin and V. M. Vinokur, Phys. Rev. Lett. 81, 3952 (1998); N. B. Kopnin, Rep. Prog. Phys. 65, 1633 (2002).

${ }^{21}$ E. B. Sonin, V. B. Geshkenbein, A. van Otterlo, and G. Blatter, Phys. Rev. B 57, 575 (1998).
${ }^{22}$ A. van Otterlo, M. Feigelman, V. Geshkenbein, and G. Blatter, Phys. Rev. Lett. 75, 3736 (1995).

${ }^{23}$ M. M. Özer, J. R. Thompson, and H. H. Weitering, Nat. Phys. 2, 173 (2006).

${ }^{24}$ L. Burlachkov, Phys. Rev. B 47, 8056 (1993).

${ }^{25}$ V. N. Kopylov, A. E. Koshelev, and I. F. Schegolov, Phys. C (Amsterdam) 170, 291 (1990).

${ }^{26}$ S.-Z. Lin, T. Nishio, L. N. Bulaevskii, M. J. Graf, and Y. Hasegawa, Phys. Rev. B 85, 134534 (2012). 